Research Article

\title{
Ultrawideband Harmonic Suppression in Microstrip Patch Antenna Using Novel Defected Ground Structures
}

\author{
Jun Fan, ${ }^{1,2}$ Jiangchuan Lin, ${ }^{1,2}$ Feng Qin $\mathbb{D}^{1,2}$ Jinliang Cai, ${ }^{1,2}$ and Mingxu Kan ${ }^{1}$ \\ ${ }^{1}$ Institute of Applied Electronics, China Academy of Engineering Physics, Mianyang 621900, China \\ ${ }^{2}$ Key Laboratory of Science and Technology on Complex Electromagnetic Environment, China Academy of Engineering Physics, \\ Mianyang 621900, China \\ Correspondence should be addressed to Feng Qin; fq_soul2000@163.com
}

Received 26 May 2020; Revised 19 September 2020; Accepted 30 September 2020; Published 14 October 2020

Academic Editor: Renato Cicchetti

Copyright (c) 2020 Jun Fan et al. This is an open access article distributed under the Creative Commons Attribution License, which permits unrestricted use, distribution, and reproduction in any medium, provided the original work is properly cited.

\begin{abstract}
Suppressing higher-order modes up to sixth harmonics of the fundamental working frequency in a single-layer microstrip line-fed patch antenna has been successfully demonstrated. Higher-order modes of the antenna are suppressed by simultaneously employing a circular head open stub connected to the feed line and a novel defected ground structure (DGS) beneath the feed line. The filtering property of the antenna has been improved by the novel DGS significantly. The role played by the novel DGS is carefully analyzed, and the effect of some key parameters of DGS is further discussed. A microstrip patch antenna with the working frequency $3.13 \mathrm{GHz}\left(f_{\mathrm{c}}\right)$ is designed and manufactured. The designed antenna possesses an ultrawideband harmonic suppression capability ranging from $2 f_{\mathrm{c}}$ to $6 f_{\mathrm{c}}$. More importantly, the area occupied by the novel DGS is decreased by $41 \%$ compared to the earlier design.
\end{abstract}

\section{Introduction}

In modern wireless communication systems, the microstrip patch antennas have been widely employed for their features of excellent polarization and easy integration with microwave integrated circuits. In these systems, the microstrip antenna acts not only as a radiator, but also as a resonator for the power amplifiers in active circuitry. However, such an antenna can produce detrimental electromagnetic radiation, affecting the performance of these systems, if it is short of proper design to suppress the harmonic resonance and spurious emission. In order to solve the problem, an additional filter is placed between the microstrip antenna and amplifier $[1,2]$. However, the fundamental operating frequency and impedance matching property of the antenna will be affected by introducing a conventional filter, which may significantly degrade the performance of the system. Moreover, the filter structure is often bulky and is not prone to integration and miniaturization.

Alternative approaches such as the introduction of photonic bandgap structures [3], tuning stubs [4-6], DGS
$[7,8]$, and hybrid structures [9-13] have been proposed to overcome the problem. In 1999, a two-dimensional photonic bandgap structure was employed by Horii and Tsutsumi to reduce the harmonic radiation in a rectangular microstrip patch antenna, and suppression up to third harmonics was demonstrated [3]. Besides, by placing tuning stub at a proper position at the feed line, the third harmonic suppression was also realized [4-6]. DGS, as another effective way to suppress the harmonic radiation in microstrip patch antenna, has become popular in recent years $[7,8]$. However, it is still difficult to achieve a higher-order harmonic suppression in the microstrip antenna by the use of one specific structure. Hybrid structures were therefore employed for improving the suppression property and up to the fourth harmonic suppression was successfully made in [11, 12]. In [11], a pair of circular headed dumbbell DGSs and a circular head open stub were used to form a low-pass filter, which finally achieved up to the fourth harmonic suppression. In [12], a pair of partial ring DGSs instead of a pair of circular headed dumbbell DGSs was utilized, lowering the DGS area by $40 \%$. Recently, a pair of I-shaped DGSs and an inverted L-shaped 
stub were proposed to realize the fifth harmonic suppression [13]. However, the area occupied by DGS is relatively large. From the viewpoint of antenna design, the physical area of DGS is also an important factor that we need to take into consideration, especially in microwave integrated circuits. Smaller DGS area can leave maximum area for integration with other devices.

This work is therefore aimed to explore an improved antenna design which possesses various outstanding features: (1) ultrawideband harmonic suppression ranging from $2 f_{\mathrm{C}}$ to $6 f_{\mathrm{C}}$ and (2) occupation area of novel DGS decreasing significantly by $41 \%$ compared with the earlier one. Moreover, suppression of radiation by $10-15 \mathrm{~dB}$ at higher-order harmonic resonances has been verified through both simulation and experiment.

\section{Design and Analysis}

The schematic diagram of the newly designed microstrip patch antenna is shown in Figure 1(a). It is a combination of three simple structures, namely, a conventional microstrip patch antenna, a circular head open stub, and a novel DGS. The geometrical parameters of the newly designed antenna are listed in Table 1 . The simulated $S_{11}$ parameters of the conventional antenna and newly designed antenna are shown in Figure 1(b). These two antennas resonate around $3.13 \mathrm{GHz}$ with their fundamental mode. The conventional antenna resonates at many other undesired modes in the frequency range from $6 \mathrm{GHz}$ to $19 \mathrm{GHz}$, including the first harmonics to the sixth harmonics $\left(\mathrm{TM}_{20}, \mathrm{TM}_{30}, \mathrm{TM}_{04}\right.$, $\mathrm{TM}_{50}$, and $\mathrm{TM}_{60}$ ) and other mixed modes. In contrast, the newly designed antenna suppresses all the higher-order modes successfully without affecting the fundamental resonance frequency. These modes have been primarily identified with the assistance of computer simulation and are shown in Figure 2. It is apparent that the fundamental mode of these two antennas is nearly the same, while the higherorder resonance modes of the newly designed antenna are suppressed successfully, which further verifies the effectiveness of the newly designed antenna.

Because the introduction of a circular head open stub and novel DGS varies the properties of the conventional patch antenna significantly, the role played by the circular head open stub and novel DGS is therefore investigated carefully. The configuration of the circular head open stub and novel DGS is shown in Figure 3(a), the circular head open stub is connected to the feed line, and the DGS, which is located beneath the feed line, is etched on the ground plane. The radius of circular stub, the distance between the center of circular stub and the edge of feed line, and the distance from the center of circular stub to the center of DGS are $r_{\mathrm{c}}, h_{\mathrm{c}}$, and $L_{\mathrm{c}}$, respectively. The simulated $\mathrm{S}_{21}$ of the circular head open stub is displayed in Figure 3(b), the stop frequency is $6.6 \mathrm{GHz}$, and the $-10 \mathrm{~dB}$ suppression bandwidth is from $5.9 \mathrm{GHz}$ to $7.47 \mathrm{GHz}$ (relative bandwidth is $23.5 \%)$. By introducing DGS, the suppression bandwidth is significantly broadened. We can clearly see from Figure 3(b) that the filtering characteristics of the configuration of circular stub with DGS is greatly improved, the $-10 \mathrm{~dB}$ suppression bandwidth is from $5.72 \mathrm{GHz}$ to $19 \mathrm{GHz}$, and the relative bandwidth is increased from $23.5 \%$ to $107.4 \%$. Moreover, the effect of $L_{\mathrm{c}}$ on the filtering property of circular stub with DGS configuration was investigated and shown in Figure 4. It is apparent that when $L_{\mathrm{c}}$ is in the range from $0.75 \mathrm{~mm}$ to $2.45 \mathrm{~mm}$, relative suppression bandwidth of $\sim 107.4 \%$ at $-10 \mathrm{~dB}$ can be obtained. In other words, the DGS can well vary the suppression property of circular stub, demonstrating outstanding and unique features.

Since the introduction of DGS can greatly improve the suppression property of the microstrip patch antenna, the novel DGS is therefore discussed in details. The DGS beneath the $50 \Omega$ microstrip feed line is depicted in Figure 5(a), which mainly consists of two concentric partial rings. The outer partial ring is generated by truncating a ring at a distance $w_{\mathrm{r}}$ from its boundary. The inner partial ring is produced by firstly truncating a ring with rectangle having width of $w_{\mathrm{s}}$ at its center, and then connecting the two partial rings by a slot having width of $d$. Other parameters include outer radius $r_{\mathrm{O}}$ and inner radius $r_{\mathrm{O}}-s$, and outer radius $r_{\mathrm{i}}$ and inner radius $r_{\mathrm{i}}-s$ for the outer partial ring and inner partial ring, respectively.

The simulated $S_{21}$ of the novel DGS is shown in Figure 5(b). The novel DGS can not only produce two transmission zeros on the filtering characteristics curve, with resonant frequencies at $6.40 \mathrm{GHz}$ and $12.08 \mathrm{GHz}$, respectively, but also possess an extremely wide $-10 \mathrm{~dB}$ suppression bandwidth ranging from $6.29 \mathrm{GHz}$ to 14.12 GHz. The wide suppression property is probably resulted from the coupling of the outer partial ring and inner partial ring. The coupling leads to the splitting of intrinsic resonance peak of the outer partial ring and inner partial ring, giving rise to an improved harmonic suppression capability. Besides, the resonant frequency of the circular head open stub is positioned in between these two resonance peaks, which can further improve the suppression property of the newly designed antenna. Compared with the most compact design reported, which are composed of two cascaded DGS sections separated by a distance, the novel DGS has more outstanding features, such as a significantly improved $-10 \mathrm{~dB}$ suppression bandwidth, a much larger energy splitting of the resonance peaks, and an even sharper cutoff response. Moreover, current distributions at the two resonance peaks of novel DGS were simulated and are displayed in Figure 5(c). It can be clearly observed that the two resonance peaks are originated from the coupling between the outer partial ring and the inner partial ring, and the energy transmitted through the feed line is successfully suppressed by the novel DGS.

The parameter $r_{\mathrm{g}}$ indicates the distance between DGS and patch edge. Smaller $r_{\mathrm{g}}$ is prone to the miniaturization design for the microstrip patch antenna, but it will affect the filtering characteristics of the antenna. As a result, the filtering property of the antenna is investigated by varying $r_{\mathrm{g}}$ in the range from $7 \mathrm{~mm}$ to $15 \mathrm{~mm}$ (Figure 6). We find that when the distance $r_{\mathrm{g}}$ is $11 \mathrm{~mm}$, i.e., $0.11 \lambda$, the $\mathrm{S}_{11}$ is larger than $-3 \mathrm{~dB}$ in the bandwidth of $6 \mathrm{GHz}$ to $19 \mathrm{GHz}$. In other words, the widest harmonic suppression bandwidth of the patch antenna is achieved as $r_{\mathrm{g}}$ equals $11 \mathrm{~mm}$. 


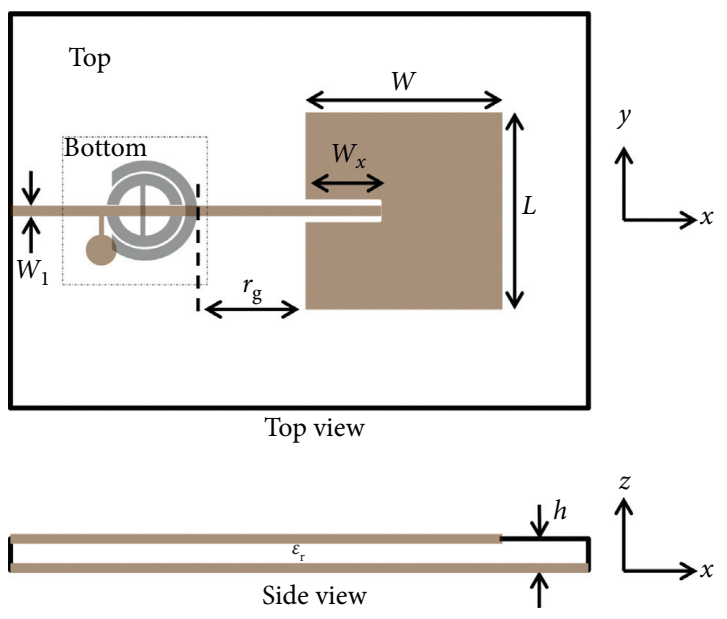

(a)

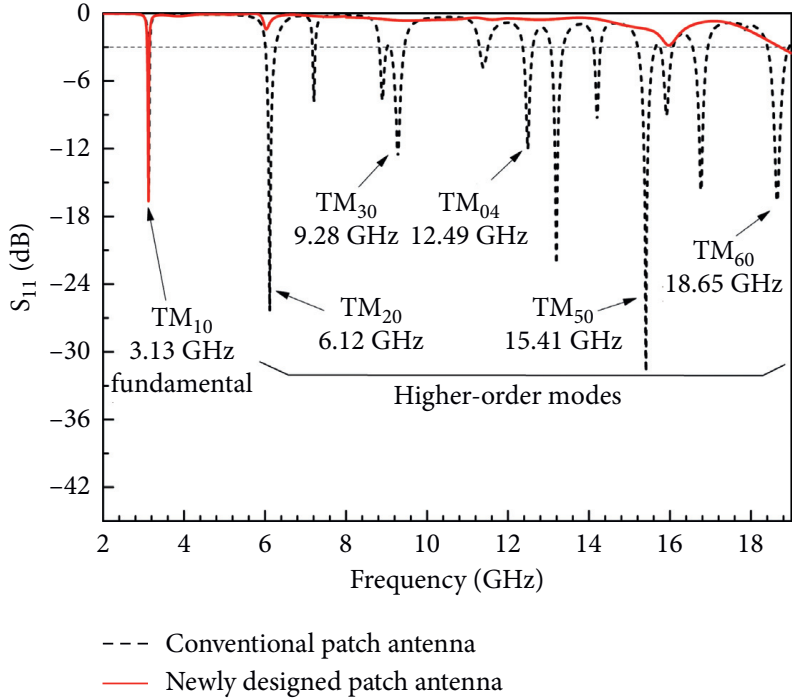

(b)

FIGURE 1: (a) Schematic illustrating the newly designed patch antenna: (b) simulated $S_{11}$ versus frequency for the conventional patch antenna and newly designed patch antenna.

TABLE 1: Geometrical parameters of the newly designed antenna.

\begin{tabular}{lc}
\hline Parameter & Value \\
\hline$W$ & $32 \mathrm{~mm}$ \\
$L$ & $32 \mathrm{~mm}$ \\
$W_{\mathrm{x}}$ & $11.6 \mathrm{~mm}$ \\
$W_{\mathrm{l}}$ & $1.6 \mathrm{~mm}$ \\
$h$ & $0.508 \mathrm{~mm}$ \\
$\varepsilon_{\mathrm{r}}$ & 2.2 \\
$r_{\mathrm{c}}$ & $2 \mathrm{~mm}$ \\
$r_{\mathrm{g}}$ & $11 \mathrm{~mm}$ \\
$h_{\mathrm{c}}$ & $1.2 \mathrm{~mm}$ \\
$L_{\mathrm{c}}$ & $1.4 \mathrm{~mm}$ \\
$r_{\mathrm{o}}$ & $2.9 \mathrm{~mm}$ \\
$r_{\mathrm{i}}$ & $2.2 \mathrm{~mm}$ \\
$s$ & $0.5 \mathrm{~mm}$ \\
$w_{\mathrm{r}}$ & $1.4 \mathrm{~mm}$ \\
$w_{\mathrm{s}}$ & $0.5 \mathrm{~mm}$ \\
$d$ & $0.15 \mathrm{~mm}$ \\
\hline
\end{tabular}

\section{Test and Results}

To examine the effectiveness of the novel DGS, a prototype of the newly designed antenna fabricated on a Rogers Duroid 5880 substrate with $\varepsilon_{\mathrm{r}}=2.2, h=0.508 \mathrm{~mm}$, and $\tan \delta=0.009$ is illustrated in Figure 7. The conventional patch antenna is also fabricated and measured for comparison. The $\mathrm{S}$ parameters were measured by using Agilent's N5222B network analyzer. The gain and radiation pattern were measured in far field by adopting an anechoic chamber.

Figure 8 shows the simulated and measured $S_{11}$ characteristics of both the conventional patch antenna and the newly designed patch antenna. The simulation matches the experiment very well for each antenna. It can be distinctly seen that the newly designed patch antenna works at its fundamental frequency of $3.13 \mathrm{GHz}$ while the higher-order modes in the frequency range from $6 \mathrm{GHz}$ to $19 \mathrm{GHz}$ are suppressed. Moreover, no noticeable variation in the impedance matching is observed at the working frequency in the newly designed antenna for the presence of the circular head open stub and novel DGS.

The radiation patterns of the conventional patch antenna and newly designed patch antenna at the working frequency $(f=3.13 \mathrm{GHz})$ are shown in Figure 9. Presence of the novel DGS has minor effect on the radiation for both the copolarized and cross-polarized fields. Measured gain at the working frequency of the newly designed antenna is $6.63 \mathrm{dBi}$, which is almost identical to that of the conventional patch antenna $(6.77 \mathrm{dBi})$.

The simulated and measured peak gains at the harmonic resonance modes of these two patch antennas are displayed in Figure 10. The newly designed antenna has successfully suppressed all the harmonic resonance modes, including the first harmonics to the sixth harmonics $\left(\mathrm{TM}_{20}, \mathrm{TM}_{30}, \mathrm{TM}_{04}\right.$, $\mathrm{TM}_{50}$, and $\mathrm{TM}_{60}$ ). 10-15 dB suppression has been demonstrated at the harmonic frequencies.

Finally, the comparison between this work and earlier designs is made, from the perspective of bandwidth of harmonic suppression and the DGS area required on the ground plane. The comparison results are listed in Table 2, which shows a very wide range of variation. In [8-10], suppression up to the third harmonics is demonstrated. Studies [11, 12] can address up to fourth harmonics, and [12] is found to be most compact. In [13], suppression up to the fifth harmonics is achieved, while the DGS area is relatively large. However, in this work, the size of DGS is further reduced by $41 \%$ compared to the most compact design reported [12]. More importantly, an ultrawideband harmonic suppression ranging from $2 f_{\mathrm{C}}$ to $6 f_{\mathrm{C}}$ is achieved by the newly designed antenna, which is the widest harmonic suppression to the best of our knowledge. 


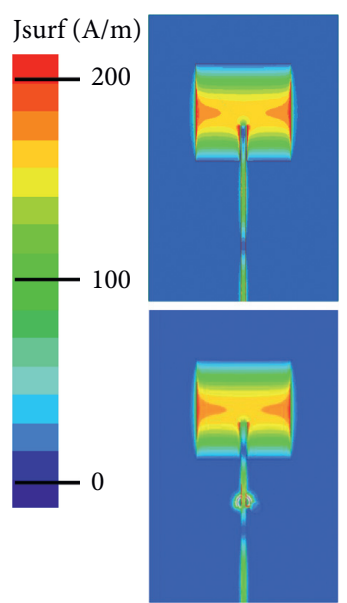

(a)
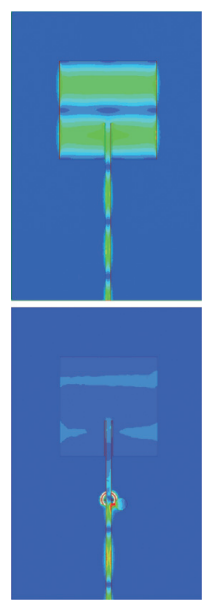

(b)
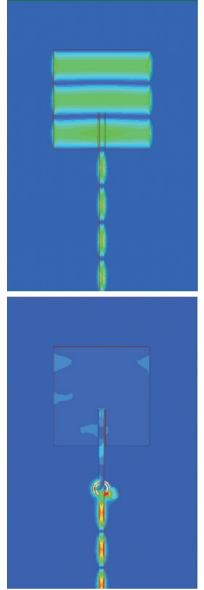

(c)
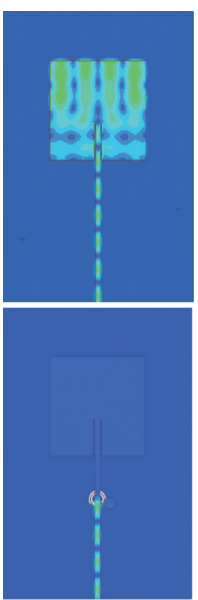

(d)
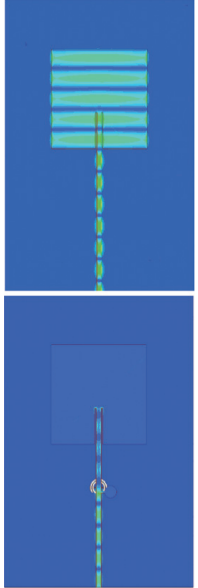

(e)

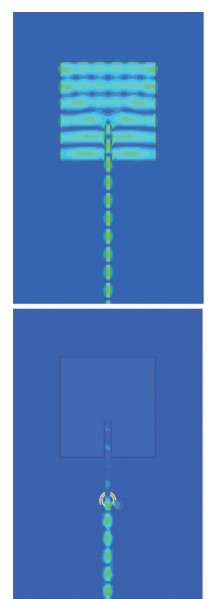

(f)

Figure 2: Simulated current distribution of the conventional patch antenna and newly designed patch antenna: (a) $\mathrm{TM}_{10}(f=3.13 \mathrm{GHz})$, (b) $\mathrm{TM}_{20}(f=6.12 \mathrm{GHz}),(\mathrm{c}) \mathrm{TM}_{30}(f=9.28 \mathrm{GHz}),(\mathrm{d}) \mathrm{TM}_{04}(f=12.49 \mathrm{GHz}),(\mathrm{e}) \mathrm{TM}_{50}(f=15.41 \mathrm{GHz})$, and (f) $\mathrm{TM}_{60}(f=18.65 \mathrm{GHz})$.

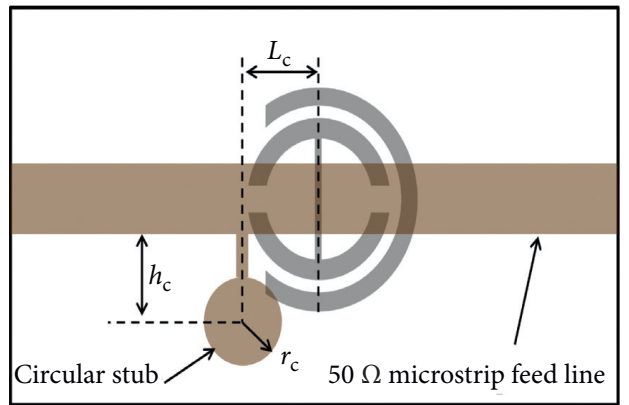

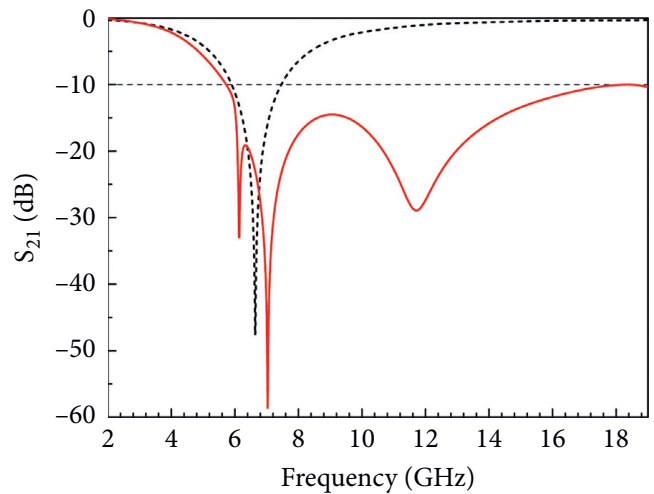

- - - Only stub

__ Stub with DGs

(b)

FIGURE 3: (a) Schematic illustrating the circular head open stub and novel DGS: (b) simulated $\mathrm{S}_{21}$ for the configurations of only circular stub and circular stub with DGS, respectively.

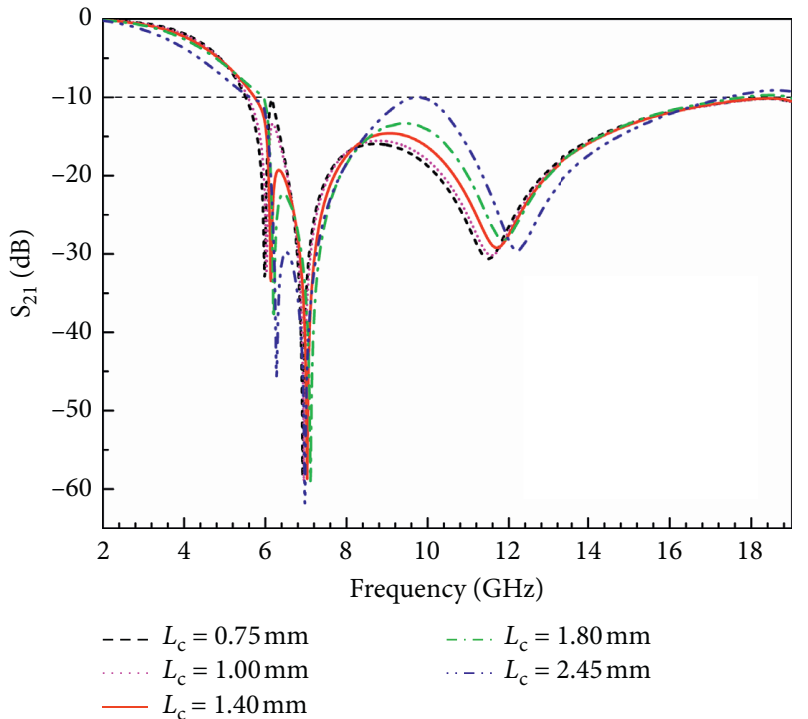

FIgURE 4: Simulated $S_{21}$ of the circular stub with DGS configuration having different $L_{\mathrm{c}}$ values. 


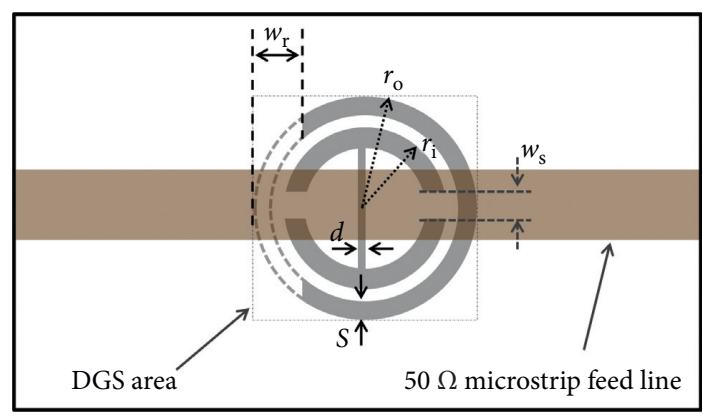

(a)

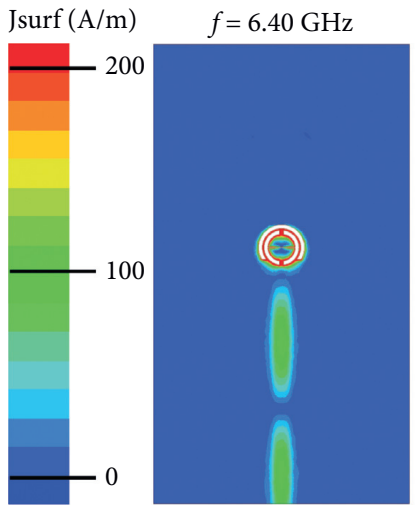

(c)

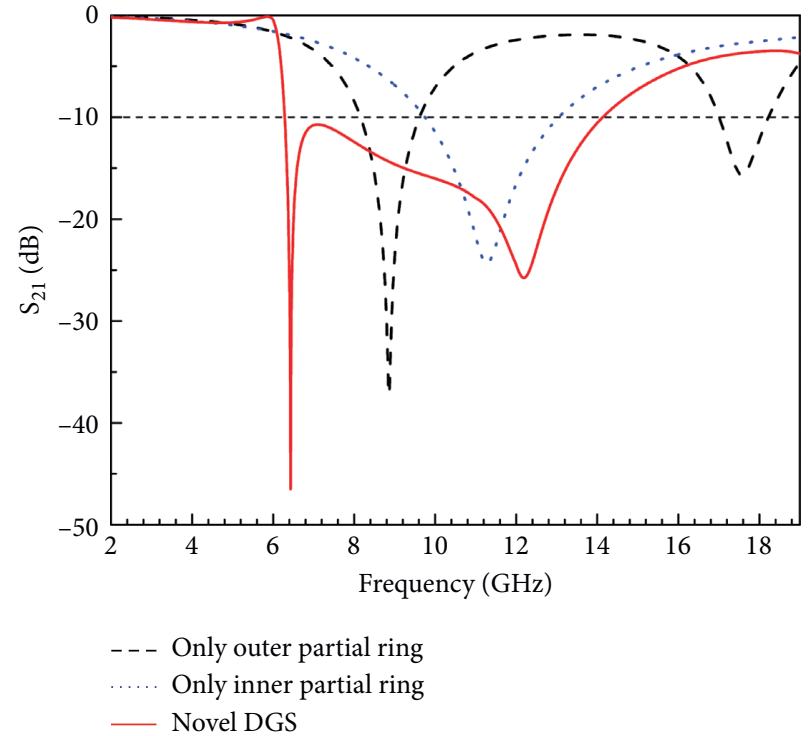

(b)

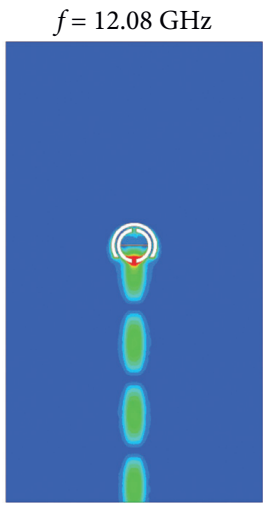

FIgURE 5: (a) Structural diagram of the novel DGS below the $50 \Omega$ microstrip feed line. (b) Simulated $S_{21}$ of the outer partial ring, inner partial ring, and novel DGS below the $50 \Omega$ microstrip feed line. (c) Simulated current distributions at the two resonance peaks of novel DGS.

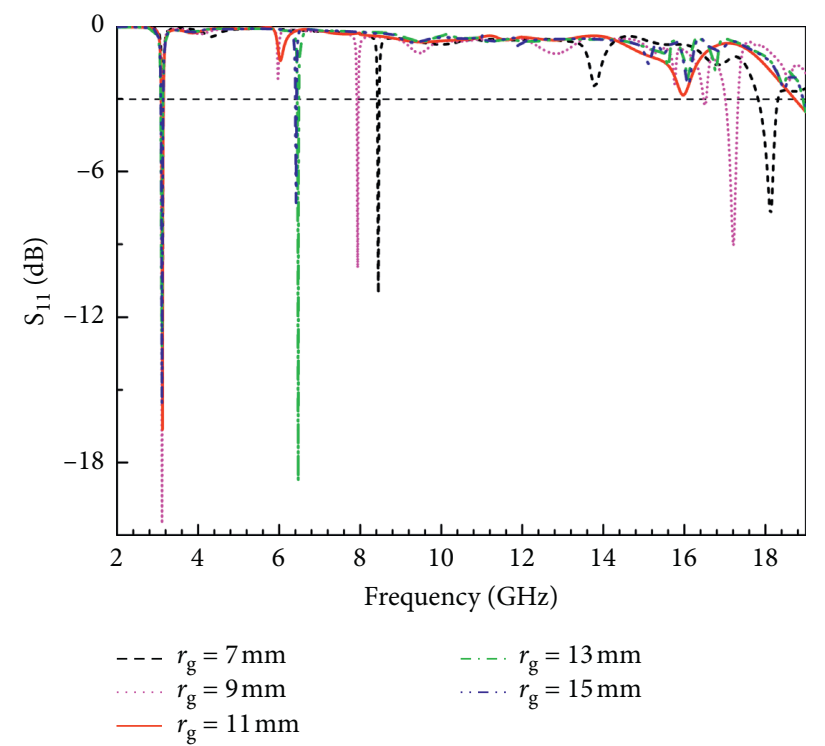

Figure 6: Simulated $S_{11}$ of newly designed patch antenna with different $r_{\mathrm{g}}$ values. 


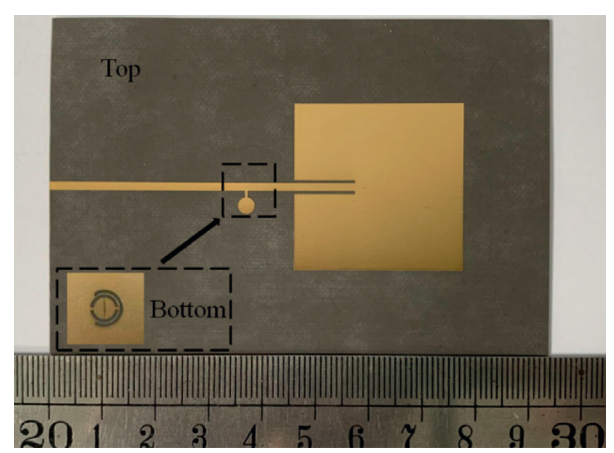

FIgURE 7: Photograph of the newly designed antenna. Inset: back view showing the novel DGS.

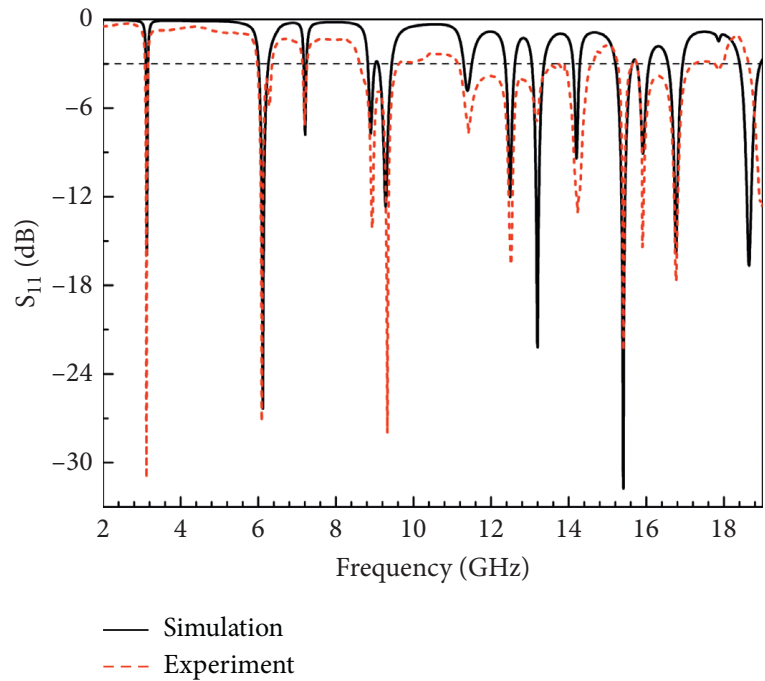

(a)

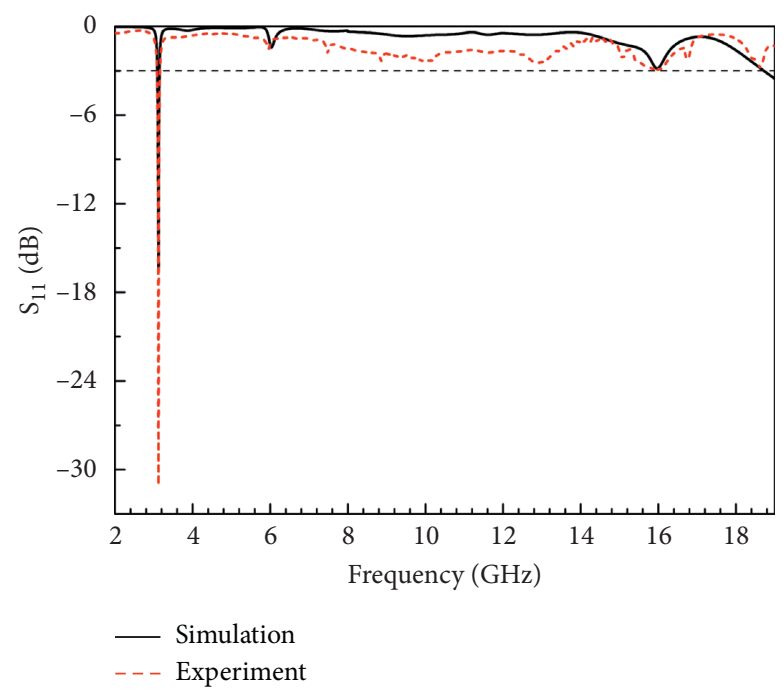

(b)

FiguRE 8: Simulated and measured $S_{11}$ of the conventional patch antenna and newly designed patch antenna: (a) conventional patch antenna; (b) newly designed patch antenna.

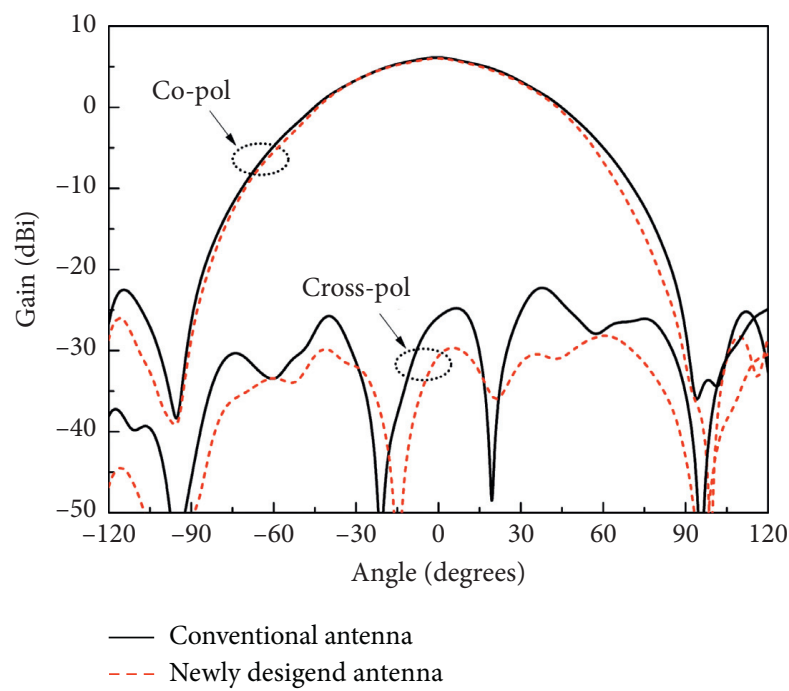

(a)

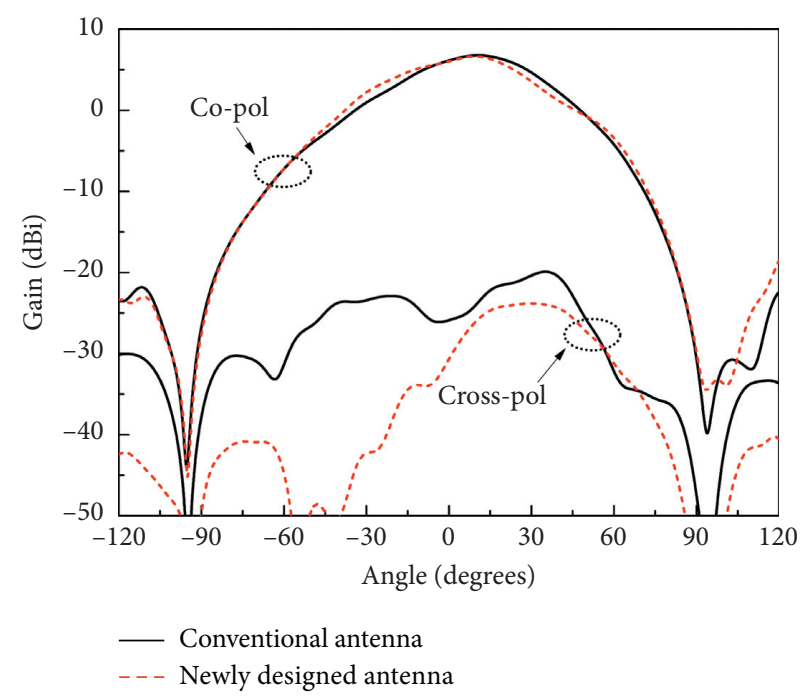

(b)

FiguRE 9: Measured radiation patterns of the conventional patch antenna and newly designed patch antenna at the fundamental frequency of $3.13 \mathrm{GHz}$ : (a) E-plane; (b) H-plane. 


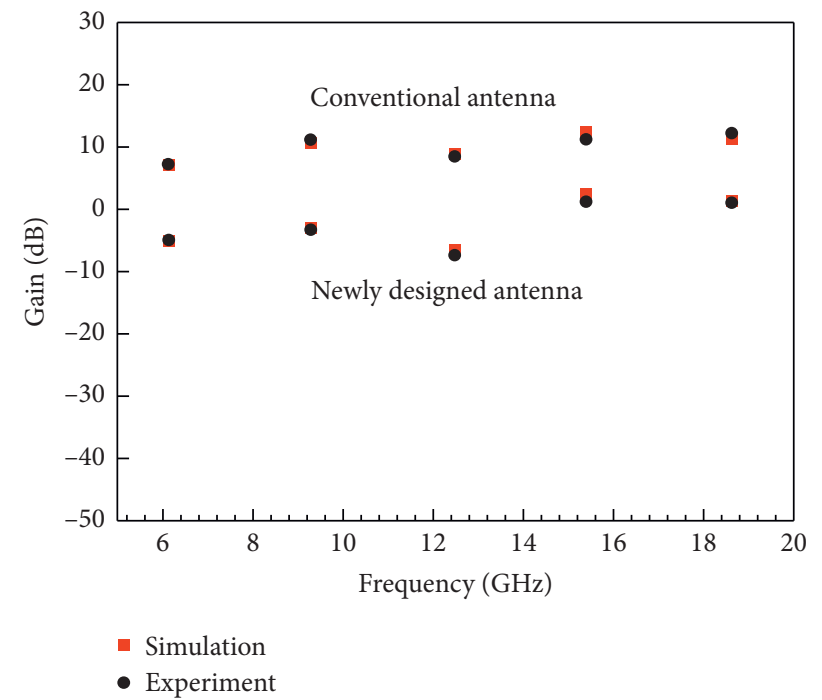

FIGURE 10: Comparison of the simulated and measured peak gains at the harmonic resonance modes of the conventional patch antenna and newly designed patch antenna.

TABLE 2: Comparison between this work and references.

\begin{tabular}{lccccc}
\hline Ref. & Fundamental freq. $(\mathrm{GHz})$ & No. of harmonics supp. & DGS used & Extracircuit & DGS area $\left(\lambda_{c}^{2} \times 10^{4}\right)$ \\
\hline$[8]$ & 5.775 & 3 & 2 square dumbbell & No & 108 \\
{$[9]$} & 1.79 & 2 & 1 square dumbbell & PBG & 120 \\
{$[10]$} & 0.9 & 3 & 2 square dumbbell & PBG & 891 \\
{$[11]$} & 3.12 & 4 & 2 circular dumbbell & Open stub & 102 \\
{$[12]$} & 3.125 & 4 & 2 partial ring & Open stub & 63 \\
{$[13]$} & 2.43 & 5 & 2 I-shaped DGS & Open stub & 149 \\
This work & 3.13 & 6 & Novel DGS & Open stub & 36 \\
\hline
\end{tabular}

\section{Conclusion}

Suppression of higher-order modes up to sixth harmonics of the fundamental working frequency has been successfully realized in a newly designed antenna. The novel DGS, employed in the antenna design, is improved significantly compared to earlier ones in terms of its compactness without compromising in performance. This would facilitate the applications of this antenna in microwave integrated circuits, particularly where a single substrate is utilized for implementing printed antennas along with active devices and circuits.

\section{Data Availability}

All data used to support the findings of this study are available from the corresponding author upon request.

\section{Conflicts of Interest}

The authors declare that there are no conflicts of interest regarding the publication of this paper.

\section{Acknowledgments}

This work was supported by the Defense Industrial Technology Development Program of China (JCKY2016212B034).

\section{References}

[1] S. Ladan, A. B. Guntupalli, and K. Wu, "A high-efficiency $24 \mathrm{GHz}$ rectenna development towards millimeter-wave energy harvesting and wireless power transmission," IEEE Transactions on Circuits and Systems I: Regular Papers, vol. 61, no. 12 , pp. 3358-3366, 2014.

[2] K. Shafique, B. A. Khawaja, M. D. Khurram et al., "Energy harvesting using a low-cost rectenna for internet of things (IoT) applications," IEEE Access, vol. 6, pp. 30932-30941, 2018.

[3] Y. Horri and M. Tsutsumi, "Harmonic control by photonic bandgap on microstrip patch antenna," IEEE Microwave and Guided Wave Letters, vol. 9, no. 1, pp. 13-15, 1999.

[4] F.-R. Hsiao, T.-W. Chiou, and K. L. Wong, "Harmonic control of a square microstrip antenna operated at the $1.8 \mathrm{GHz}$ band," in Proceedings of the Asia-Pacific Microwave Conference, pp. 1052-1055, Taipei, Taiwan, 2001.

[5] C. K. Ghosh, "Harmonics suppression of microstrip antenna using open ended stubs," Microwave and Optical Technology Letters, vol. 58, no. 6, pp. 1340-1345, 2016.

[6] S.-Y. Lin, K.-C. Huang, and J.-S. Chen, "Harmonic control for an integrated microstrip antenna with loaded transmission line," Microwave and Optical Technology Letters, vol. 44, no. 4, pp. 379-383, 2005.

[7] Y. J. Sung, M. Kim, and Y. S. Kim, "Harmonics reduction with defected ground structure for a microstrip patch antenna," IEEE Antennas and Wireless Propagation Letters, vol. 2, pp. 111-113, 2003. 
[8] I. Chang and B. Lee, "Design of defected ground structures for harmonic control of active microstrip antenna," Proceedings of the IEEE Antennas and Propagation Society International Symposium, vol. 2, pp. 852-855, 2002.

[9] Y. J. Sung and Y.-S. Kim, "An improved design of microstrip patch antennas using photonic bandgap structure," IEEE Transactions on Antennas and Propagation, vol. 53, no. 5, pp. 1799-1804, 2005.

[10] H. Liu, Z. Li, X. Sun, and J. Mao, "Harmonic suppression with photonic bandgap and defected ground structure for a microstrip patch antenna," IEEE Microwave and Wireless Components Letters, vol. 15, no. 2, pp. 55-56, 2005.

[11] M. K. Mandal, P. Mondal, S. Sanyal, and A. Chakrabarty, "An improved design of harmonic suppression for microstrip patch antennas," Microwave and Optical Technology Letters, vol. 49, no. 1, pp. 103-105, 2007.

[12] S. Biswas, D. Guha, and C. Kumar, "Control of higher harmonics and their radiations in microstrip antennas using compact defected ground structures," IEEE Transactions on Antennas and Propagation, vol. 61, no. 6, pp. 3349-3353, 2013.

[13] J. Acharjee, K. Mandal, S. K. mandal, and P. P. Sarkar, "Suppressing up to fourth harmonic of an ISM band microstrip patch antenna using compact defected ground structures," Microwave and Optical Technology Letters, vol. 59, no. 9, pp. 2254-2259, 2017. 